\title{
Transmission of Cytauxzoon felis to domestic cats by Amblyomma americanum nymphs
}

\author{
Kelly E. Allen*, Jennifer E. Thomas, Megan L. Wohltjen and Mason V. Reichard
}

\begin{abstract}
Background: Successful Cytauxzoon felis transmission studies have occurred using Amblyomma americanum adults acquisition-fed as nymphs on an experimentally infected domestic cat or Dermacentor variabilis adults fed as nymphs on a splenectomized bobcat. Here, we evaluated $A$. americanum and D. variabilis nymphs acquisition-fed as larvae on a C. felis-infected carrier domestic cat for competence to transmit the protozoan parasite as nymphs to naive, healthy domestic cats.

Methods: Amblyomma americanum and D. variabilis larvae were applied to a chronically infected, parasitemic C. felis donor cat (Felis catus) and allowed to feed to repletion. Engorged larvae were collected and held through ecdysis. Three cats were each infested with $66 \mathrm{~A}$. americanum or $66 \mathrm{D}$. variabilis emerged nymphs. Cytauxzoon felis infections in principal cats were determined by clinical signs and detection of circulating parasite by blood smear and PCR evaluation.

Results: Clinical signs of cytauxzoonosis were observed in cats infested with A. americanum nymphs beginning 12-15 days post-infestation (dpi). The same cats were PCR positive on 12-14 dpi; piroplasms were evident in blood smears at $16 \mathrm{dpi}$, and macrophage schizonts were observed in stained spleen impression smears in two animals at necropsy. Cats infested with acquisition-fed D. variabilis nymphs remained clinically normal and did not develop detectable parasitemia over the course of the study as determined by blood smear and PCR.

Conclusions: Cytauxzoon felis was successfully transmitted to domestic cats by A. americanum nymphs acquisition-fed as larvae on the donor cat. However, we were not able to transmit $C$. felis to healthy domestic cats with $D$. variabilis nymphs that were simultaneously acquisition-fed on the same donor cat. Results from this study suggest that larval and nymphal A. americanum likely play important roles in natural transmission cycles of $C$. felis.
\end{abstract}

Keywords: Amblyomma americanum, Cytauxzoon felis, Dermacentor variabilis, Ecdysis, Larvae, Nymphs, Piroplasm, Schizont, Sporozoite, Transstadial

\section{Background}

Cytauxzoon felis is a blood-borne apicomplexan infecting domestic and wild felids in the southeastern, south-central, and mid-Atlantic USA [1-3]. The disease associated with C. felis infection in domestic cats, cytauxzoonosis or bobcat fever, is severe. Salient clinical features of the acute syndrome include an abrupt onset of pyrexia, listlessness, anemia, inappetence, icterus, and dyspnea, with death usually occurring approximately one week after presentation $[2,4,5]$. Survivor domestic cats have been documented and

\footnotetext{
* Correspondence: Kelly.allen10@okstate.edu

Department of Veterinary Pathobiology, Oklahoma State University Center for Veterinary Health Sciences, Stillwater, OK 74078, USA
}

are thought to remain persistently infected with piroplasms in circulation $[4,6-8]$.

The natural route of $C$. felis transmission to felid intermediate hosts occurs via the blood-feeding of infected ticks harboring parasite sporozoites $[9,10]$. Dermacentor variabilis was initially accepted to be the natural tick vector of $C$. felis based on its presence in some enzootic areas and experiments using splenectomized wild felids [10-13]. More recent experiments documented repeat transstadial transmission of $C$. felis from survivor domestic cats to naive, immunocompetent cats by Amblyomma americanum $[9,10]$.

(c) The Author(s). 2019 Open Access This article is distributed under the terms of the Creative Commons Attribution 4.0 International License (http://creativecommons.org/licenses/by/4.0/), which permits unrestricted use, distribution, and reproduction in any medium, provided you give appropriate credit to the original author(s) and the source, provide a link to the Creative Commons license, and indicate if changes were made. The Creative Commons Public Domain Dedication waiver (http://creativecommons.org/publicdomain/zero/1.0/) applies to the data made available in this article, unless otherwise stated. 
In studies surveying wild-caught $A$. americanum, molecular evidence of $C$. felis infection has been detected in $0.0 \%, 1.5 \%$, or $15.3 \%$ of adult ticks sampled and in $0.8 \%$ of nymphs sampled $[1,9,14]$. Despite undetectable to low infection prevalence in field-collected $A$. americanum populations in some areas, confirmed experimental data as well as geographical and seasonal trends of cytauxzoonosis cases point to $A$. americanum as the primary definitive host and tick vector of $C$. felis $[3,9,10]$.

Previous C. felis transmission experiments have utilized adult ticks acquisition-fed as nymphs on donor felids $[9,10,12,13]$. The present study evaluated the ability of $A$. americanum and $D$. variabilis larvae to acquire $C$. felis from an experimentally infected, parasitemic donor cat and transmit the parasite as nymphs to naïve healthy cats as determined by clinical signs and parasitemia in infested principals.

\section{Methods}

\section{Animals and housing}

Cats were housed individually in cages and maintained indoors in a climate-controlled room with a 12-h light: 12 -h dark photocycle. Animals were provided food and water ad libitum.

The C. felis donor cat was a cytauxzoonosis survivor animal from a previous tick transmission study [9] and was used to infect larval ticks. Six domestic cats (Aa-1, Aa-2, Aa-3, Dv-1, Dv-2 and Dv-3) served as principals for C. felis transmission by nymphs acquisition-fed as larvae. All principals were approximately six months of age and weighed between $2.5-3.2 \mathrm{~kg}$ at the time of arrival. Cats were vaccinated against feline rhinotracheitis virus, calicivirus, panleukopenia virus, and rabies virus prior to arrival.

During tick infestation experiments, cats were housed in metabolic cages over metal catch pans of water lined along the edges with double-sided tape in a sealed, climate-controlled room at OSU's Center for Veterinary Health Sciences (Stillwater, OK, USA) [9, 10].

\section{Acquisition feeding of tick larvae on the $C$. felis donor cat} The donor cat was infested with $A$. americanum and $D$. variabilis larvae hatched from approximately one-half of an egg clutch from females of each species purchased from the National Tick Rearing Lab at Oklahoma State University (Stillwater, OK, USA). Tick species propagated in this laboratory are considered pathogen-free and are therefore widely used by researchers conducting transmission experiments $[10,15]$.

Prior to infestation, whole blood in EDTA was collected from the $C$. felis donor cat to confirm the presence of piroplasms in erythrocytes in a Wright-Giemsa stained blood film. The donor cat was anesthetized immediately prior to infestation via intramuscular injection with $4.0 \mathrm{mg} / \mathrm{kg}$ tiletamine and zolazepam (Telazol'; Zoetis, Parsippany, NJ, USA). Larval ticks were applied beneath a Surgi-Sox (DogLeggs, Reston, VA, USA) wrap that covered the donor cat's thorax and abdomen. The metal catch pan directly beneath the cage and the water moat below were checked daily for replete larvae. PCR was not performed on individual or pooled replete tick larvae, as positive results would have been attributable to $C$. felis piroplasms imbibed from the donor cat, rather than established parasite infections within removed ticks.

\section{Tick maintenance through ecdysis}

Replete larvae acquisition-fed on the $C$. felis donor cat were collected daily and placed in paper cartons that were stored long-term in a humidity chamber (90-98\% constant humidity) at $25{ }^{\circ} \mathrm{C}$ with a 14-h light-dark photophase. Larvae were held through ecdysis, and emerged nymphs were used to infest separate principal cats in the C. felis transmission experiments approximately four to six weeks after molting.

Prevalence of $C$. felis infection in emerged A. americanum or $D$. variabilis cohorts was not determined by PCR prior to infestation of principal cats in an effort to preserve potentially infected ticks for transmission experiments. Also, the number of organisms present in the molted nymphs may have been below the level of detection by the PCR assay utilized, even with multiple nymphs being pooled into small groups.

\section{Cytauxzoon felis transmission with emerged nymphs}

To confirm that principal cats were not infected with $C$. felis prior to study commencement, whole blood was evaluated for circulating parasite by blood smear examination and PCR. Two separate experiments were conducted to assess $C$. felis transmission to cats using $D$. variabilis and $A$. americanum nymphs that were acquisition-fed as larvae on the donor cat. The numbers of molted nymphs used in the transmission experiments were divided such that each principal within a group (A. americanum $v s D$. variabilis) would receive an equal number of ticks. Nymphs of each species $(n=66)$ were applied to separate naïve cats and allowed to feed to repletion.

In the first transmission experiment, cats Aa-1 and Aa-2 were infested with $A$. americanum nymphs and cats $\mathrm{Dv}-1$ and $\mathrm{Dv}-2$ were infested with $D$. variabilis nymphs. In the second experiment, cat Aa- 3 was infested with $A$. americanum nymphs and cat Dv-3 was infested with $D$. variabilis nymphs. Nymphs fed to repletion on cat principals were collected and stored at $-20{ }^{\circ} \mathrm{C}$ in ethanol for later DNA extraction and PCR analysis.

\section{Determination of $C$. felis infection in principal cats}

Principal cats were observed daily for clinical signs of cytauxzoonosis by physical examination, rectal temperatures, and 
weights. Additionally, whole blood in EDTA was aseptically collected via venipuncture of jugular or cephalic veins from cats at various intervals throughout the study $(0,2,5,7,8,9$, $10,12,14$ and 16 days post-infestation) for microscopic examination of Wright-Giemsa stained blood films and DNA extraction for PCR.

Cats with C. felis parasitemia were euthanatized via intravenous administration of Beuthanasia-D $(0.20 \mathrm{ml} / \mathrm{kg}$; Schering-Plough, Summit, NJ, USA) after piroplasms were detected in blood and/or after they began showing clinical signs consistent with cytauxzoonosis such as fever, anorexia and lethargy. Cats that did not become infected with $C$. felis were euthanatized or transferred to another protocol.

\section{DNA extraction and PCR analysis}

Total genomic DNA was isolated from $200 \mu \mathrm{l}$ of whole blood using the QIAamp ${ }^{\circledR}$ DNA Mini Kit (Qiagen, Valencia, CA, USA) per the manufacturer's instructions. Separate pools of five $A$. americanum and $D$. variabilis replete nymphs that had been stored at $-20{ }^{\circ} \mathrm{C}$ in ethanol were extracted for DNA as previously described $[9,10]$.

Primers C. felis I/II forward and C. felis II reverse and nested primers CfnestF and CfnestR were used to amplify a 289 bp region of the $18 S$ rRNA gene of $C$. felis from blood and tick extracts $[9,10]$. PCR products were separated in a $1.5 \%$ agarose matrix stained with ethidium bromide and viewed with ultraviolet light.

\section{Results}

\section{Confirmation of $C$. felis in donor cat and infestation with larvae}

The donor cat was subclinical and had a parasitemia of $0.004 \%$ at the time of larvae acquisition feeding as determined by blood film evaluation for piroplasms in erythrocytes. After infestation, as many as possible replete larvae of each tick species were collected (not enumerated) from the donor cat.

\section{Infestation of principal cats with molted nymphs}

The minimum number of acquisition-fed $A$. americanum or D. variabilis needed to experimentally transmit $C$. felis to naïve cats is not known, and is likely multi-factorial. In previous experiments, 50-100 A. americanum adults and 50-95 D. variabilis adults acquisition-fed as nymphs were applied to principal cats; only $A$. americanum infestations resulted in successful $C$. felis infection of principal cats [9, 10, 16]. A total of $230 \mathrm{~A}$. americanum and $220 \mathrm{D}$. variabilis molted nymphs were recovered from the donor cat infestation experiment in this study.

Sixty-six molted nymphs were applied to each principal cat in the first transmission experiment; 29 and 9 replete $A$. americanum nymphs were collected from cats Aa-1 and Aa-2, respectively, and 23 and 32 replete $D$. variabilis nymphs were collected from cats Dv-1 and Dv-2, respectively. In the second experiment, 22 replete $A$. americanum and 22 replete $D$. variabilis nymphs were recovered from cats Aa-3 and Dv-3, respectively. Ticks applied during the infestations but not accounted for at times of collection presumably escaped from Surgi-Sox wraps on principal cats and were groomed off or were trapped in double-sided tape or water moats.

\section{Clinical signs of $C$. felis infection in principal cats}

Cats infested with acquisition-fed $A$. americanum nymphs (Aa-1, Aa-2 and Aa-3) developed clinical signs consistent with cytauxzoonosis, whereas cats infested with acquisition-fed D. variabilis nymphs (Dv-1, Dv-2 and Dv-3) did not exhibit clinical signs of cytauxzoonosis at any point during the study. Cat Aa-1 had an elevated body temperature beginning on $14 \mathrm{dpi}$ infestation. Lethargy was observed on $15 \mathrm{dpi}$ and enlarged popliteal lymph nodes were detected on exam on $16 \mathrm{dpi}$. Cat Aa-2 began losing weight at $12 \mathrm{dpi}$ and maintaining an elevated body temperature on $13 \mathrm{dpi}$. This cat was observed to be lethargic at $15 \mathrm{dpi}$ and slight splenomegaly was evident upon abdominal palpation the following day. Cat Aa-3 was consistently pyrexic and losing weight beginning $12 \mathrm{dpi}$ and was noted to be depressed and lethargic on $14 \mathrm{dpi}$. Splenomegaly and hepatomegaly was noted at $15 \mathrm{dpi}$, and the animal was euthanatized shortly thereafter.

Cats Dv-1 and Dv-2 continued to be monitored by temperature and general health observations until 27 dpi; both cats maintained normal body temperatures and appeared clinically healthy during this time. Cat Dv-3 was monitored for general overall health, was PCR negative on $14 \mathrm{dpi}$, and was euthanatized at the same time as cat Aa-3.

\section{Assessment of circulating C. felis in principal cats after infestation with nymphs}

In previous studies, circulating parasite was detected by PCR and blood smear in experimentally infected cats infested with $A$. americanum by $8-18 \mathrm{dpi}$ and $18 \mathrm{dpi}$, respectively; time to parasite detection in circulation for cats experimentally infested with $D$. variabilis is unknown, although splenectomized cats died of cytauxzoonosis 13-17 dpi after adult tick engorgement in one study $[10,13,16]$.

In the present study, blood sampling of principal cats was ceased once both clinical signs consistent with cytauxzoonosis and observation of parasite on blood films occurred for A. americanum-infested cats. Results of blood smear and PCR evaluation of principal cats are summarized in Table 1. Cats Aa-1 and Aa-2 (infested with $A$. americanum) were PCR positive on 12,14 , and $16 \mathrm{dpi}$; piroplasms were observed in stained blood films at 16 dpi. Cytauxzoon felis schizonts were observed in 
Table 1 PCR results for C. felis infection in principal cats after infestation with A. americanum or D. variabilis nymphs

\begin{tabular}{|c|c|c|c|c|c|c|c|c|c|c|c|c|}
\hline \multirow{3}{*}{$\begin{array}{l}\text { Days post- } \\
\text { infestation }\end{array}$} & \multicolumn{6}{|c|}{ Cats infested with nymphal A. americanum } & \multicolumn{6}{|c|}{ Cats infested with nymphal D. variabilis } \\
\hline & \multicolumn{2}{|c|}{ Cat Aa-1 } & \multicolumn{2}{|c|}{ Cat Aa-2 } & \multicolumn{2}{|c|}{ Cat Aa-3 } & \multicolumn{2}{|c|}{ Cat Dv-1 } & \multicolumn{2}{|c|}{ Cat Dv-2 } & \multicolumn{2}{|c|}{ Cat Dv-3 } \\
\hline & PCR & Smear & PCR & Smear & PCR & Smear & PCR & Smear & PCR & Smear & $\mathrm{PCR}$ & Smear \\
\hline 0 & - & - & - & - & - & - & - & - & - & - & - & - \\
\hline 2 & - & - & - & - & - & - & - & - & - & - & - & - \\
\hline 5 & - & - & - & - & ns & ns & - & - & - & - & ns & ns \\
\hline 7 & - & - & - & - & ns & ns & - & - & - & - & ns & ns \\
\hline 8 & ns & ns & ns & ns & - & - & ns & ns & ns & ns & - & - \\
\hline 9 & - & - & - & - & ns & ns & - & - & - & - & ns & ns \\
\hline 10 & ns & ns & ns & ns & - & - & ns & ns & ns & ns & - & - \\
\hline 12 & + & - & + & - & ns & ns & - & - & - & - & ns & ns \\
\hline 14 & + & - & + & - & + & - & - & - & - & - & - & - \\
\hline 16 & + & + & + & + & + & - & - & - & - & - & ns & ns \\
\hline
\end{tabular}

Abbreviation: ns, no sample collected or tested

spleen impression smears at the time of necropsy. Cat Aa-3, also infested with A. americanum and PCR positive on 14 and $16 \mathrm{dpi}$, was euthanatized.

Blood smear and PCR results remained negative for cats infested with $D$. variabilis throughout the study. Although DV-3 was euthanatized after only 16 days of PCR and blood smear monitoring, cats Dv-1 and Dv-2 were monitored by body temperature for $27 \mathrm{dpi}$, and for general overall health for $35 \mathrm{dpi}$. On $35 \mathrm{dpi}$, cat Dv-1 was euthanatized and cat Dv-2 was transferred to another protocol to serve as a negative control animal for C. felis cell culture propagation, before which PCR screening of the animal was conducted to confirm lack of $C$. felis infection.

\section{PCR of replete nymphs for detection of $C$. felis DNA}

A single pool of replete nymphs of each tick species was tested for $C$. felis infection by PCR. DNA extracts from separate pools of five replete $A$. americanum and $D$. variabilis nymphs fed on cat principals (held at $-20{ }^{\circ} \mathrm{C}$ in ethanol) tested negative for C. felis DNA. The number of C. felis organisms within tick sample pools may have been too low for detection by the PCR method utilized. Xenodiagnostic evidence indicated that a necessary proportion of feeding $A$. americanum were infected with parasite, while this was not the case for feeding $D$. variabilis in this study.

\section{Discussion}

The results of the present study further substantiate $A$. americanum as the predominant natural tick vector in C. felis transmission cycles involving domestic cats. Previous studies evaluated $C$. felis transmission to domestic cats using $D$. variabilis and $A$. americanum adults acquisition-fed as nymphs on carrier felids $[9,10,12,13]$. The present study documented transstadial transmission of C. felis in A. americanum from larvae to nymphs, and the competence of emerged nymphs to transmit parasite to healthy domestic cats. Nymphal D. variabilis, simultaneously acquisition-fed as larvae on the same carrier animal at the time, did not transmit C. felis to principal cats.

The inability of $D$. variabilis nymphs to transmit $C$. felis to naïve cats in these experiments may have been influenced by several factors. First, genetic variability of C. felis strain internal transcribed spacer (ITS) regions in domestic cats has been documented [2]. The C. felis genotype maintained in our experimental donor cats is well-adapted to $A$. americanum based on repeat successful transmission studies with adult ticks, while attempts utilizing $D$. variabilis adults with this strain have failed $[9,10]$. Other C. felis strains in nature may be more evolutionarily adapted to successful transmission by $D$. variabilis. It is also possible that natural $D$. variabilis populations have a different microbiome than colony reared cohorts which better supports the transstadial maintenance of $C$. felis and subsequent transmission of parasite to felid hosts.

Also, the infection prevalence of $C$. felis between the two tick species could have differed during blood feeding on the donor cat, despite being acquisition-fed on the cat at the same time [9]. Amblyomma americanum larvae may have acquired more piroplasms than did $D$. variabilis larvae. As a result, fewer viable sporozoites were present in the salivary glands of molted $D$. variabilis nymphs than in $A$. americanum nymphs, thereby reducing the likelihood of $C$. felis transmission by $D$. variabilis. The relatively low level of parasitemia ( $0.004 \%$ of erythrocytes with piroplasms) in the experimentally infected carrier cat may have also influenced the numbers of viable sporozoites available within ticks after molting. Dermacentor variabilis larvae may require a higher number of $C$. felis piroplasms relative to $A$. americanum to maintain enough parasites through the molt to transmit sporozoites during the nymphal stage. As 
a result, more $D$. variabilis nymphs than $A$. americanum may be needed to successfully transmit $C$. felis to naïve, immunocompetent cats. Time from nymphal emergence to next blood-meal acquisition may also differently affect $C$. felis maintenance and subsequent salivarian transmission between the two tick species.

Previous successful experiments with $D$. variabilis used 400 adults to transmit $C$. felis to immunosuppressed bobcats [12]. Successful experiments with $A$. americanum have been performed using 25 pairs of adult males and females to immunocompetent domestic cats $[9,10]$. Here, as few as $33 \mathrm{~A}$. americanum nymphs were able to transmit C. felis to healthy cats, although not all ticks applied may have been infected; $C$. felis transmission by the same number of $D$. variabilis nymphs did not occur based on absence of both clinical signs and detectable parasitemia in infested cat principals.

We did not detect $C$. felis DNA in nymphal pool DNA extracts of either tick species, but it is possible that the C. felis infection prevalence of feeding nymphal cohorts was low, and because of this the few replete ticks randomly pooled for testing were not infected with parasite. It is also possible that organism number between pooled ticks may have been below the detection limit of the PCR assay utilized, especially considering the low level of parasitemia in the donor cat upon which the ticks fed during the previous instar.
In field studies, pools of wild-caught adult $D$. variabilis and $A$. americanum nymphs and adults have tested positive for $C$. felis DNA by PCR, but infection prevalence of C. felis in either tick species in nature is highly variable and may be influenced by geographical region. Surveys by different research groups investigating $C$. felis infection prevalence in adult ticks indicate molecular evidence of infection in $0-15.4 \%$ of $A$. americanum sampled and in 0 $15.9 \%$ of $D$. variabilis sampled. Cytauxzoon felis has been detected by PCR in $0-0.8 \%$ of wild-caught $A$. americanum nymphs surveyed; parasite prevalence in wild-caught $D$. variabilis nymphs has not been documented [9, 17-19]. In these instances, PCR-positive ticks collected in the field may have recently fed on parasitemic hosts, or ticks could have taken a blood meal during previous instars from felid hosts with high enough parasitemia to enable detection of C. felis DNA within ticks after ecdysis $[9,20]$.

Lastly, it is possible that molecular and clinical evidence of $C$. felis infection in cats infested with infected $D$. variabilis may be delayed in comparison to cats infested with infected $A$. americanum. However, although the third clinically normal cat infested with $D$. variabilis nymphs (Dv-3) was euthanatized after only $16 \mathrm{dpi}$, the first two cats infested with $D$. variabilis nymphs were monitored daily by body temperature for $27 \mathrm{dpi}$ and either euthanatized (Dv-1) or used as a negative control blood source in a cell culture study (Dv-2). The transitioned cat (Dv-2) was screened by

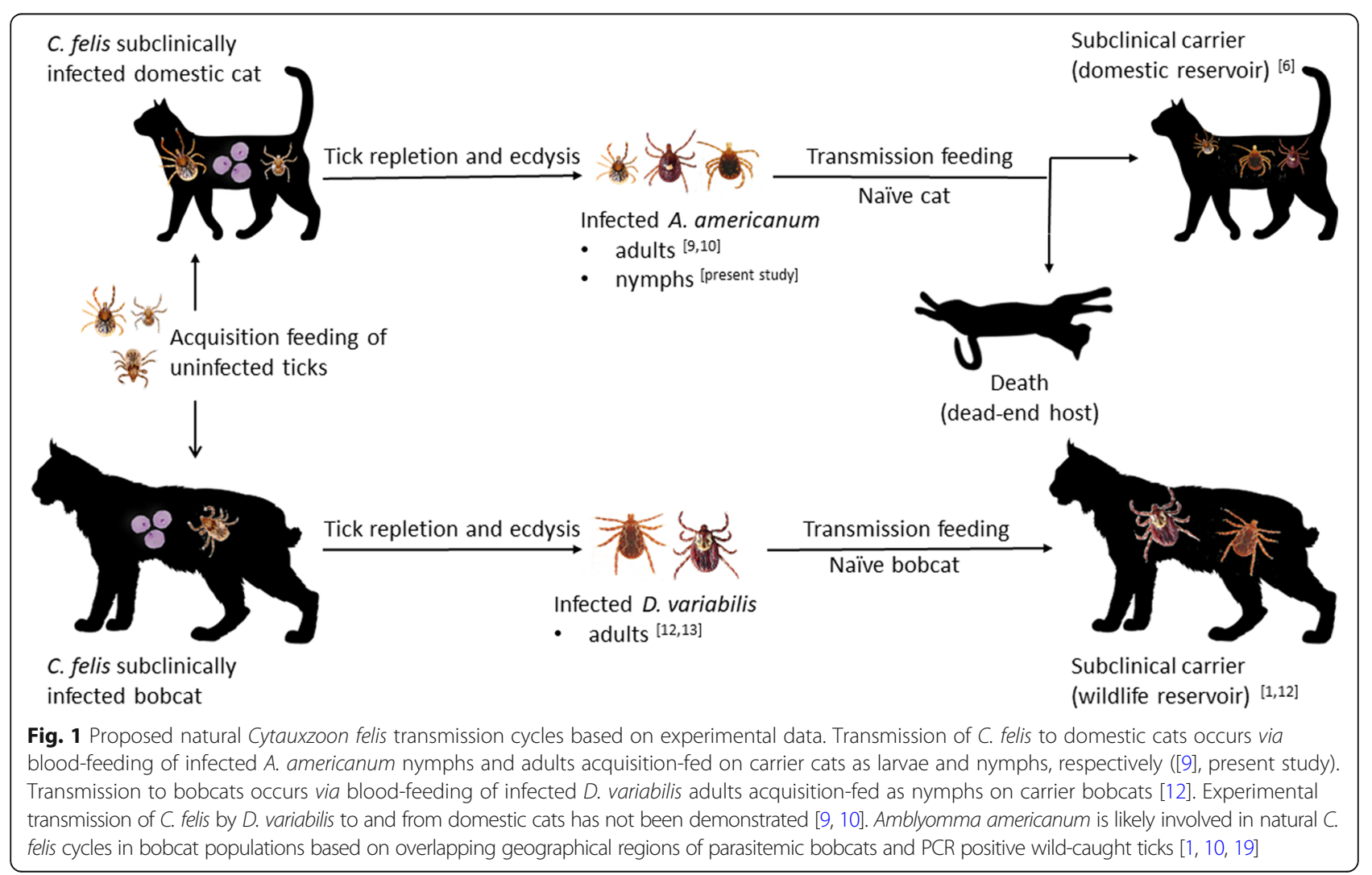


PCR to confirm negative $C$. felis status, which it remained. The authors acknowledge that xenodiagnosis may have been a useful approach to further to rule out $C$. felis infection in acquisition-fed ticks, but did not have the opportunity to conduct these experiments.

\section{Conclusions}

Previously it was shown that feeding A. americanum adults, infected with $C$. felis as nymphs, can transmit the parasite to naïve cats. Recent surveys have established that domestic cats are natural hosts of larval, nymphal and adult $A$. americanum [17-19]. Here we demonstrate that feeding $A$. americanum nymphs, infected with $C$. felis as larvae, can transmit the parasite to naïve felid hosts in nature as depicted in Fig. 1. Nymphal A. americanum may be an under recognized source of $C$. felis infection to domestic cats.

\section{Acknowledgements}

The authors would like to thank Laboratory Animal Resources personnel at Oklahoma State University Center for Veterinary Health Sciences for their assistance with donor and principal cat husbandry.

\section{Funding}

The studies were funded by Oklahoma State University Center for Veterinary Health Sciences.

\section{Availability of data and materials}

The data supporting the conclusions of this article are included within the article. Raw data from the article are kept on file in the KEA laboratory and is available upon request for review by bona fide researchers.

\section{Authors' contributions}

KEA, JET, MLW and MVR participated in the study design and completion of work, and were involved in drafting the manuscript. All authors read and approved the final version of the manuscript.

\section{Ethics approval}

The study was governed by an Animal Care and Use Protocol (ACUP) approved by the Oklahoma State University Institutional Animal Care and Use Committee (IACUC). Experiments involving the donor and principal domestic cats were conducted under an ACUP approved by the IACUC at Oklahoma State University.

\section{Consent for publication}

Not applicable.

\section{Competing interests}

The authors declare that they have no competing interests.

\section{Publisher's Note}

Springer Nature remains neutral with regard to jurisdictional claims in published maps and institutional affiliations.

Received: 29 August 2018 Accepted: 20 December 2018

Published online: 11 January 2019

\section{References}

1. Shock BC, Murphy SM, Patton LL, Shock PM, Olfenbuttel C, Beringer J, et al. Distribution and prevalence of Cytauxzoon felis in bobcats (Lynx rufus), the natural reservoir, and other wild felids in thirteen states. Vet Parasitol. 2011;175:325-30.

2. Brown HM, Lockhart JM, Latimer KS, Peterson DS. Identification and genetic characterization of Cytauxzoon felis in asymptomatic domestic cats and bobcats. Vet Parasitol. 2010;172:311-6.
3. Reichard MV, Baum KA, Cadenhead SC, Snider TA. Temporal occurrence and environmental risk factors associated with cytauxzoonosis in domestic cats. Vet Parasitol. 2008;152:314-20.

4. Nagamori Y, Slovak JE, Reichard MV. Prevalence of Cytauxzoon felis infection in healthy free-roaming cats in north-central Oklahoma and central lowa. JFMS Open Rep. 2016;2:2055116916655174.

5. Meinkoth $\mathrm{JH}$, Kocan AA. Feline cytauxzoonosis. Vet Clin North Am Small Anim Pract. 2005;35:89-101.

6. Rizzi TE, Reichard MV, Cohn LA, Birkenheuer AJ, Taylor JD, Meinkoth JH. Prevalence of Cytauxzoon felis infection in healthy cats from enzootic areas in Arkansas, Missouri, and Oklahoma. Parasit Vectors. 2015:8:13.

7. Brown HM, Latimer KS, Erikson LE, Cashwell ME, Britt JO, Peterson DS. Detection of persistent Cytauxzoon felis infection by polymerase chain reaction in three asymptomatic domestic cats. J Vet Diagn Invest. 2008;20: 485-8.

8. Haber MD, Tucker MD, Marr HS, Levy JK, Burgess J, Lappin MR, et al. The detection of Cytauxzoon felis in apparently healthy free-roaming cats in the USA. Vet Parasitol. 2007;146:316-20.

9. Reichard MV, Edwards AC, Meinkoth JH, Snider RA, Meinkoth KR, Heinz RE, et al. Confirmation of Amblyomma americanum (Acari: Ixodidae) as vector for Cytauxzoon felis (piroplasmorida: Theileriidae) to domestic cats. J Med Entomol. 2010:47:890-6.

10. Reichard MV, Meinkoth JH, Edwards AC, Snider TA, Kocan KM, Blouin EF, et al. Transmission of Cytauxzoon felis to a domestic cat by Amblyomma americanum. Vet Parasitol. 2009;161:110-5.

11. Birkenheuer AJ, Marr HS, Warren C, Acton AE, Mucker EM, Humphreys JG, et al. Cytauxzoon felis infections are present in bobcats (Lynx rufus) in a region where cytauxzoonosis is not recognized in domestic cats. Vet Parasitol. 2008;153:126-30.

12. Blouin EF, Kocan AA, Kocan KM, Hair J. Evidence of a limited schizogonous cycle for Cytauxzoon felis in bobcats following exposure to infected ticks. J Wildl Dis. 1987;23:499-501.

13. Blouin EF, Kocan AA, Glenn BL, Kocan KM, Hair JA. Transmission of Cytauxzoon felis Kier, 1979 from bobcats, Felis rufus (Schreber), to domestic cats by Dermacentor variabilis (Say). J Wildl Dis. 1984;20:241-2.

14. Zieman EA, Jiménez FA, Nielsen CK. Concurrent examination of bobcats and ticks reveals high prevalence of Cytauxzoon felis in southern Illinois. J Parasitol. 2017;103:343-8

15. Kocan KM, de la Fuente J, Coburn LA. Insights into the development of Ixodes scapularis: a resource for research on a medically important tick species. Parasit Vectors. 2015:8:592.

16. Thomas JE, Ohmes CM, Payton ME, Hostetler JA, Reichard MV. Minimum transmission time of Cytauxzoon felis by Amblyomma americanum to domestic cats in relation to duration of infestation, and investigation of ingestion of infected ticks as a potential route of transmission. J Feline Med Surg. 2018:20:66-72.

17. Little SE, Barrett AW, Nagamori Y, Herrin BH, Normile D, Heaney K, et al. Ticks from cats in the United States: patterns of infestation and infection with pathogens. Vet Parasitol. 2018;257:15-20.

18. Thomas JE, Staubus L, Goolsby JL, Reichard MV. Ectoparasites of freeroaming domestic cats in the central United States. Vet Parasitol. 2016;228:17-22.

19. Bondy PJ, Cohn LA, Tyler JW, Marsh AE. Polymerase chain reaction detection of Cytauxzoon felis from field-collected ticks and sequence analysis of the small subunit and internal transcribed spacer 1 region of the ribosomal RNA gene. J Parasitol. 2005;91:458-61.

20. Shock BC, Moncayo A, Cohen S, Mitchell EA, Williamson PC, Lopez G, et al. Diversity of piroplasms detected in blood-fed and questing ticks from several states in the United States. Ticks Tick Borne Dis. 2014:5:373-80. 